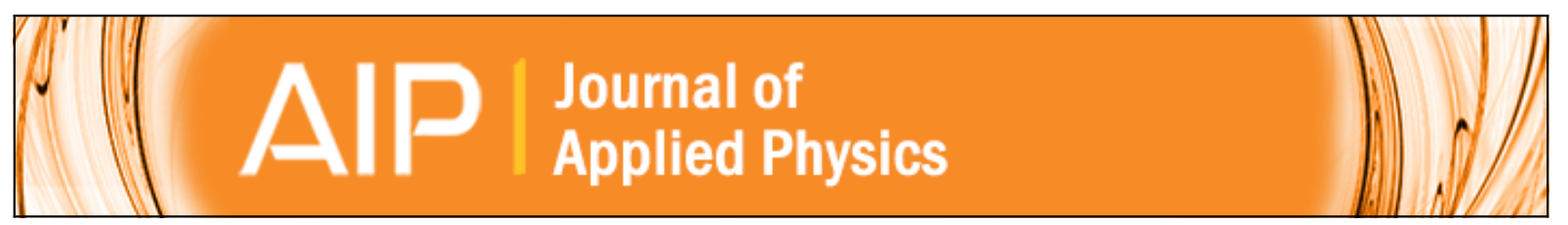

\title{
Rotatable anisotropy driven training effects in exchange biased $\mathrm{Co} / \mathrm{CoO}$ films
}

T. Dias, E. Menéndez, H. Liu, C. Van Haesendonck, A. Vantomme, K. Temst, J. E. Schmidt, R. Giulian, and J. Geshev

Citation: Journal of Applied Physics 115, 243903 (2014); doi: 10.1063/1.4885157

View online: http://dx.doi.org/10.1063/1.4885157

View Table of Contents: http://scitation.aip.org/content/aip/journal/jap/115/24?ver=pdfcov

Published by the AIP Publishing

\section{Articles you may be interested in}

The asymmetric magnetization reversal in exchange biased granular $\mathrm{Co} / \mathrm{CoO}$ films

Appl. Phys. Lett. 104, 182403 (2014); 10.1063/1.4875594

Domain formation in exchange biased $\mathrm{Co} / \mathrm{CoO}$ bilayers

J. Appl. Phys. 93, 7726 (2003); 10.1063/1.1540152

Unidirectional coercivity enhancement in exchange-biased $\mathrm{Co} / \mathrm{CoO}$

Appl. Phys. Lett. 81, 1270 (2002); 10.1063/1.1498505

Determination of exchange anisotropy by means of ac susceptometry in $\mathrm{Co} / \mathrm{CoO}$ bilayers

J. Appl. Phys. 81, 5003 (1997); 10.1063/1.364967

Measurement of the exchange anisotropy in $\mathrm{Co} / \mathrm{CoO}$ bilayers using the anisotropic magnetoresistance J. Appl. Phys. 81, 5002 (1997); 10.1063/1.364966

\section{AIP $\mid$ chaos CALL FOR APPLICANTS Seeking new Editor-in-Chief}




\title{
Rotatable anisotropy driven training effects in exchange biased $\mathrm{Co} / \mathrm{CoO}$ films
}

\author{
T. Dias, ${ }^{1,2}$ E. Menéndez, ${ }^{2}$ H. Liu, ${ }^{3}$ C. Van Haesendonck, ${ }^{3}$ A. Vantomme, ${ }^{2}$ K. Temst,${ }^{2}$ \\ J. E. Schmidt, ${ }^{1}$ R. Giulian, ${ }^{1}$ and J. Geshev ${ }^{1}$ \\ ${ }_{1}^{1}$ Instituto de Física, Universidade Federal do Rio Grande do Sul (UFRGS), RS, 91501-970 Porto Alegre, \\ Brazil \\ ${ }^{2}$ KU Leuven, Instituut voor Kern- en Stralingsfysica, Celestijnenlaan 200 D, 3001 Leuven, Belgium \\ ${ }^{3}$ KU Leuven, Laboratorium voor Vaste-Stoffysica en Magnetisme, Celestijnenlaan 200 D, 3001 Leuven, \\ Belgium
}

(Received 31 May 2014; accepted 13 June 2014; published online 25 June 2014)

\begin{abstract}
The training effect for exchange bias in field-cooled $\mathrm{Co} / \mathrm{CoO}$ bilayers films is investigated. Previous experiments on the same system have shown that, starting from the ascending branch of the first hysteresis loop, coherent magnetization rotation is the dominant reversal mechanism. This is confirmed by the performed numerical simulations, which also indicate that the training is predominantly caused by changes of the rotatable anisotropy parameters of uncompensated spins at the $\mathrm{Co} / \mathrm{CoO}$ interface. Moreover, in contrast with what is commonly assumed, the exchange coupling between the rotatable spins and the ferromagnetic layer is stronger than the coupling between the ferromagnet and the spins responsible for the bias. Thus, uncompensated spins strongly coupled to the ferromagnet contribute to the coercivity rather than to the bias, whatever the strength of their magnetic anisotropy. (C) 2014 AIP Publishing LLC.
\end{abstract}

[http://dx.doi.org/10.1063/1.4885157]

\section{INTRODUCTION}

Exchange bias $(\mathrm{EB})^{1-3}$ results from the magnetic coupling between a ferromagnet (FM) and uncompensated spins (UCSs) at the interface with an adjacent antiferromagnet (AFM). Since its discovery in $1956,{ }^{1}$ EB has been extensively studied in different types of systems including core/ shell particles, ${ }^{4}$ thin bilayer and multilayer films, and FM nanoparticles embedded in an AFM matrix. ${ }^{5}$ The bestknown manifestation of EB is the hysteresis loop shift along the field axis, referred to as the EB field $\left(H_{E B}\right)$, typically accompanied by an increase of the coercive field $\left(H_{C}\right)$ compared to that of the FM alone. Usually, EB is established by some post-deposition treatment performed under an applied magnetic field. ${ }^{2,3}$ The sample is either cooled down starting from a temperature $(T)$ higher than the blocking temperature ( $T_{B}$, above which EB vanishes) to $T<T_{B}$, or irradiated with light-ion beams. ${ }^{6,7}$ Application of a very strong magnetic field (either during the sample production or at $T$ lower than $T_{B}$ (Refs. 8 and 9) or even the FM remanent magnetization acquired prior to cooling ${ }^{10}$ can also set the effect.

An interesting phenomenon related to EB is the training effect, i.e., the frequently observed decrease of $H_{C}$ and $\left|H_{E B}\right|$ upon consecutive hysteresis loop measurements. It was first observed in $\mathrm{Co} / \mathrm{CoO}$ and $\mathrm{NiFe} / \mathrm{AFM}$ systems, ${ }^{11}$ and, since then, several models have been proposed to explain this behavior. Two types of training effects can be considered, namely athermal and thermal. While the thermal training ceases to exist at low temperatures and usually brings about small changes of $H_{C}$, the athermal training, characterized by an abrupt suppression of $H_{C}$ and $\left|H_{E B}\right|$ between the first and the second consecutively measured loops, is practically independent of temperature. ${ }^{12,13}$ Hoffmann ${ }^{14}$ proposed that the athermal training effect occurs due to a spin-flop-like coupling and that the initial spin configuration cannot be restored. Considering a single FM domain, exchange-coupled to a large number of AFM grains with threefold anisotropy, Brems et al. ${ }^{15}$ have found training due to magnetization reorientation towards easy axes far from the one closest to the direction of the cooling field and that, by applying an orthogonal in-plane magnetic field, the untrained state can be largely restored. Harres and Geshev have shown ${ }^{16}$ that athermal training effects may result from exchange and/or dipolar interactions between the FM and interface UCSs, regardless of their anisotropy type.

Several mechanisms have been proposed as being responsible for the thermal training effect. Née ${ }^{17}$ argued that the AFM coupling between first-neighbor domains caused by atomic steps on AFM interfaces could lead to thermal training. Binek assumed that the spin structure of the FM/AFM interface deviates from its equilibrium configuration, implying the decrease of $\left|H_{E B}\right|$ after each hysteresis loop trace is a consequence of thermodynamics governed spin rearrangement. ${ }^{18}$ Suess et al. ${ }^{19}$ have shown that a weak spin-flop coupling between randomly oriented AFM grains at compensated interfaces could result in EB and training. Biternas et al. ${ }^{12,20}$ using the domain-state model for $\mathrm{EB},{ }^{21}$ have estimated that the AFM spin structure rearrangement and changes of the population of the stable and unstable AFM spins during reversal are responsible for the training.

In this work, we examine, through a model for polycrystalline exchange-coupled systems with granular interfaces, low-temperature experimental magnetization data obtained on $\mathrm{Co} / \mathrm{CoO}$ bilayers that exhibit training. We found that in our films, contrary to what is commonly assumed, the exchange coupling between the rotatable UCSs and the FM 
is stronger than the coupling between the FM and the biasing UCSs, and also that the training is predominantly caused by changes of the rotatable anisotropy parameters.

\section{EXPERIMENTAL DETAILS}

A polycrystalline $30 \mathrm{~nm}$ thick Co film was grown by molecular beam epitaxy onto a thermally-oxidized $\mathrm{Si}(100)$ substrate which was first covered with a $10 \mathrm{~nm}$ thick Au buffer layer. The latter was introduced to minimize atomic intermixing between $\mathrm{Co}$ and the $450 \mathrm{~nm}$ thick $\mathrm{SiO}_{2}$, which would eventually yield unintended $\mathrm{CoO}$ formation. Both layers were deposited in ultra-high vacuum $\left(3 \times 10^{-10}\right.$ millibar) at room temperature. The Co layer consists of a mixture of face-centered cubic Co and hexagonal close-packed Co environments and stacking faults. X-ray reflectometry reveals the formation of an approximately $3 \mathrm{~nm}$ thick polycrystalline $\mathrm{CoO}$ layer formed by exposing the sample to a pure oxygen atmosphere $\left(5 \times 10^{-4}\right.$ millibar $)$ for $5 \mathrm{~min} .^{22}$

The magnetic characterization was performed by means of in-plane hysteresis loop measurements using a Quantum Design MPMS-XL7 Superconducting Quantum Interference Device magnetometer in longitudinal configuration. EB was induced by field-cooling the sample from room temperature down to the measurement temperature of $10 \mathrm{~K}$ with an applied magnetic field of $4 \mathrm{kOe}$ parallel to the sample plane. The training effect was investigated by magnetically cycling the sample until equilibrium was reached. The first four easy-axis hysteresis loops (traced with measurement magnetic field applied along the induced EB direction) together with the eighth one obtained at $10 \mathrm{~K}$ are plotted in Fig. 1.

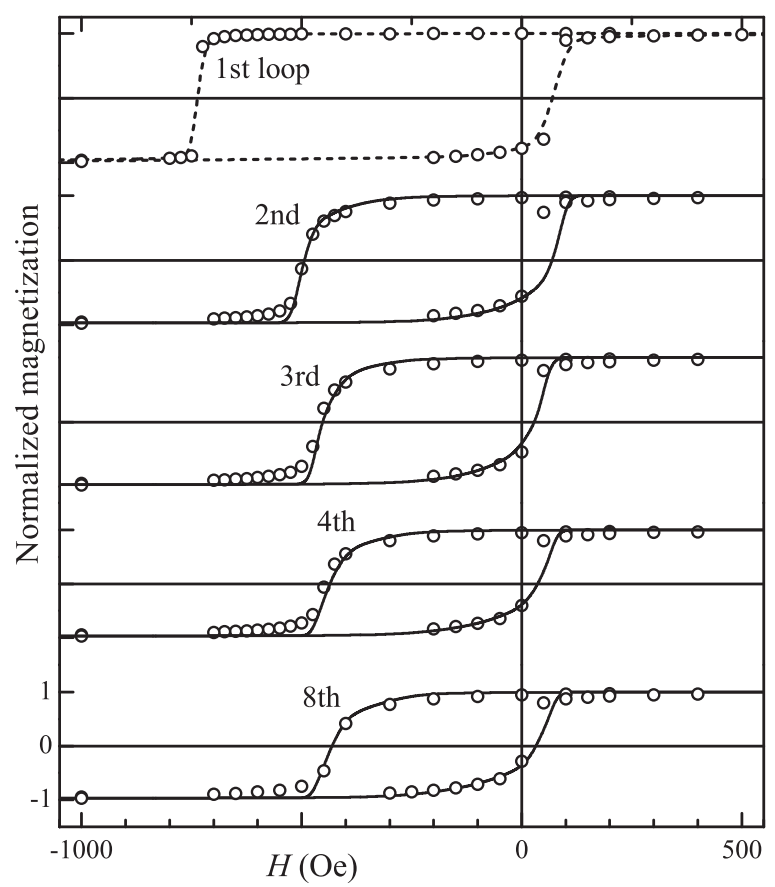

FIG. 1. The first four and the eighth easy-axis hysteresis loops of the $\mathrm{Co} /$ $\mathrm{CoO}$ bilayer at $10 \mathrm{~K}$. The symbols represent the experimental data, and the solid lines correspond to simulations performed using $2 K_{F M} / M_{F M}=100 \mathrm{Oe}$, $V / v_{i}=20, M_{F M} / m_{i}=3.1$, and a Gaussian easy-axis distribution centered at $\phi_{0}=\phi_{i, 0}=0^{\circ}$, where $i$ denotes either set or rot; $t_{i} K_{i}, J_{i}, \sigma_{\text {set }}\left(=\sigma_{F M}\right)$, and $\sigma_{\text {rot }}$ for $n$ varying between 2 and 9 are given in Fig. 2. The dashed line is a guide for the eye.
Anisotropic magnetoresistance (AMR) measurements were performed at $10 \mathrm{~K}$ using the four-probe method after the same field-cooling procedure with DC current parallel to the thus induced EB direction.

\section{MODEL}

It is currently accepted ${ }^{23-25}$ that at the FM/AFM interfaces of polycrystalline bilayers there are UCSs which behave as single FM domains and interact with the adjacent FM and/ or interfacial AFM spins. Stable UCSs do no rotate irreversibly and add to the bias (from now on, the corresponding quantities will be indexed by set). Other less-stable UCSs, due to sufficiently strong exchange interaction with the FM, rotate almost simultaneously with its magnetization, $\mathbf{M}_{\mathbf{F M}}$, and promote the $H_{C}$ enhancement. UCSs of the latter type are considered to be responsible for the Rotatable Anisotropy (RA) sensed by the FM and, in what follows, will be referred to as rot. In model calculations, RA has been initially taken into account ${ }^{26}$ by including a unidirectional RA term in the energy expression, aiming to explain the frequently observed isotropic shift of the angular variation of the ferromagnetic resonance field. Later on, when irreversible magnetization processes have been considered in order to reproduce both branches of a magnetization hysteresis loop, ${ }^{27}$ a uniaxial RA term proportional to $-\left(\mathbf{M}_{\mathbf{F M}} \cdot \mathbf{H}\right)^{2}$ has been employed ( $\mathbf{H}$ is the applied magnetic field), given that the coupling with the 'rotatable' UCSs is sensed by the FM as an additional uniaxial anisotropy with symmetry axis parallel to $\mathbf{H}$.

Numerical simulations were performed through a model for the magnetic behavior of polycrystalline FM/ AFM systems with granular interfaces, ${ }^{23}$ which accounts for the athermal training. ${ }^{16}$ The model assumes that, in accordance with the findings of $\mathrm{Li}$ and Zhang, ${ }^{28}$ for sufficiently strong FM/AFM coupling, the AFM breaks the adjacent FM into small-sized domains which interact with set- and rot-type UCSs. Different from the previous RA models, there are two terms corresponding to effective RA in the energy expression of this model, i.e., one that refers to the magnetic anisotropy of the rotatable spins and another term accounting for their exchange coupling to the FM. It is worth noting that, in polycrystalline EB systems, the frequently observed non-zero hard-axis $H_{C}$, though not well understood and often ignored, is naturally reproduced by this model.

Let us briefly sketch a straightforward version of the model. ${ }^{23}$ It considers the particular case of uniaxial in-plane anisotropies of the FM and of the UCSs and neglects the Zeeman terms of the latter, since these are usually much smaller than the Zeeman energy of the FM. Interactions between UCSs are not considered either. Therefore, the system consists of magnetic triplets (and not an FM coupled to a large numbers of AFM grains as considered in the classical model of Fulcomer and Charap ${ }^{29}$ ), each of these formed by an FM grain with volume $V$, exchange-coupled to two (a setand a rot-type) grains with UCSs, with magnetizations $\mathbf{m}_{\text {set }}$ and $\mathbf{m}_{\text {rot }}$ and volumes $v_{\text {set }}$ and $v_{\text {rot }}$, respectively. For an inplane $\mathbf{H}$, the variable part of the free energy of a triplet is 


$$
\begin{aligned}
E= & V K_{F M} \sin ^{2}\left(\phi-\phi_{0}\right)-V H M_{F M} \cos \left(\phi_{H}-\phi\right) \\
& +\sum_{i=1}^{2}\left[v_{i} K_{i} \sin ^{2}\left(\phi_{i}-\phi_{i, 0}\right)-a_{i} J_{i} \cos \left(\phi_{i}-\phi\right)\right] .
\end{aligned}
$$

Here, $K_{F M}$ and $K_{i}$ are the FM and UCS anisotropy constants, respectively, $\phi_{0}$ and $\phi_{i, 0}$ are the angles that the FM and UCS easy axes make with the EB direction of the system, and $\phi$ and $\phi_{i}$ are the angles that $\mathbf{M}_{\mathbf{F M}}$ and $\mathbf{m}_{\mathbf{i}}$ form with the EB direction; $a_{i}\left(=v_{i} / t_{i}\right)$ represents the contact area at the FM/UCS interface. The subscript $i$ denotes either set or rot-type UCS, which depends on both $K_{i}$ and the exchange coupling constants $J_{i}$, as will be discussed below. The first two terms of the above equation represent the FM's anisotropy and Zeeman energy terms, the third corresponds to the UCS's anisotropy, and the last term is the FM/UCS exchange coupling energy. The magnetization of the system is obtained by minimizing the free energy with respect to $\phi$ and $\phi_{i}$ keeping all other constants as free parameters. Our model differs from that used to explain the athermal training in $\mathrm{Co}(20 \mathrm{~nm}) / \mathrm{CoO}$ films ${ }^{30}$ since uniaxial instead of threefold anisotropy of the UCSs is considered, the FM's anisotropy has not been neglected, and the system consists of many, distributed in size and orientation, magnetic triplets (an FM grain coupled to a set- and a rot-type UCSs) instead of one FM moment coupled to a large numbers of AFM grains.

The use of uniaxial anisotropy is justified since phenomenological computations have shown that non-interacting single-domain AFMs can induce competition between magnetocrystalline (cubic) and shape (uniaxial) anisotropies. ${ }^{31}$ In the particular case of $\mathrm{CoO}$, neutron powder diffraction ${ }^{32}$ and high-resolution synchrotron powder diffraction ${ }^{33}$ have shown that distortions in crystalline structure provoke either cubic-to-tetragonal or cubic-to-monoclinic transitions at low temperature, which are accompanied by magnetostriction that leads to uniaxial anisotropy. A recent study on $3 d$-transition metal monoxides has revealed, by means of densityfunctional theory and local spin-density approximation in first-principle calculations, that those distortions of the unit cell as well as dipole-dipole interaction are responsible for the existence of only one in-plane magnetic easy axis. ${ }^{34}$

In addition to the hysteresis loop fittings, we performed AMR simulations using the same model. For the AMR, the change of resistance, $R$, as a function of the angle, $\phi_{\mathrm{M}}$, between the current direction and the magnetization, can be expressed as

$$
R\left(\phi_{\mathrm{M}}\right)=R_{\perp}+\Delta R_{0} \cos ^{2} \phi_{\mathrm{M}} .
$$

Here, $R_{\|}$and $R_{\perp}$ are the resistance for the current parallel and perpendicular to the magnetization, respectively, and $\Delta R_{0}=R_{\|}-R_{\perp}$. Easy-axis AMR fitting curves were calculated by using the above equation, where the orientation of the FM magnetization was calculated for each fixed field using the same parameters as employed for the corresponding magnetization curve simulations.

\section{RESULTS AND DISCUSSION}

Figure 2 demonstrates that $\left|H_{E B}\right|$ gradually decreases from 330 to 210 Oe between the first and the fourth loop.

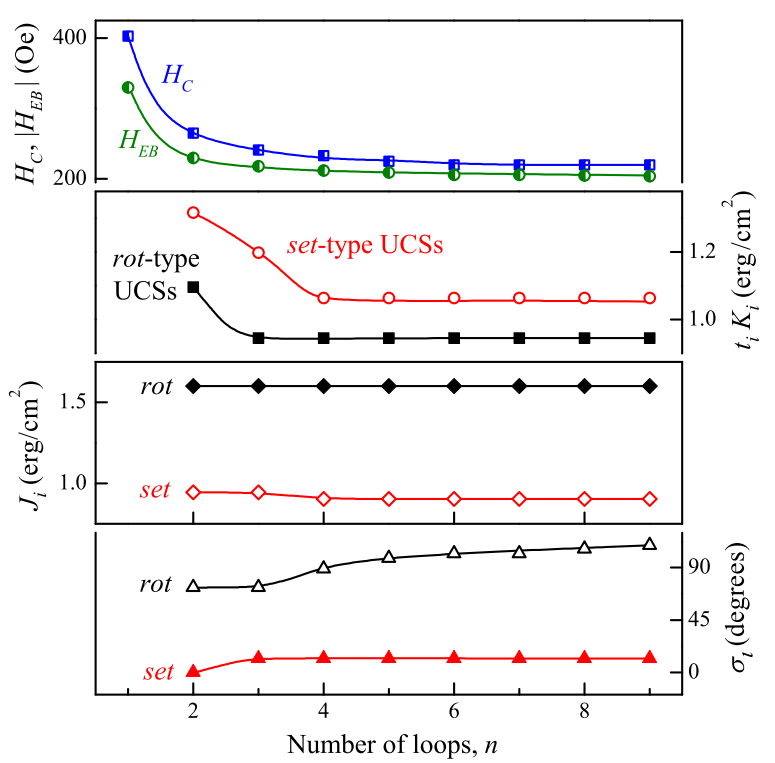

FIG. 2. $\left|H_{E B}\right|, H_{C}$ as well as the coupling and anisotropy parameters versus $n$ employed in the simulations of the fitting curves plotted in Fig. 1 (the subscript $i$ denotes either set or rot). The lines are guides to the eye.

The corresponding drop in $H_{C}$ is around $190 \mathrm{Oe}$, and, after the fourth loop, further changes are negligible. While a $\mathrm{Co}(20 \mathrm{~nm}) / \mathrm{CoO}(2 \mathrm{~nm})$ bilayer sputtered onto an oxidized $\mathrm{Si}$ wafer mainly shows athermal training only, ${ }^{15,30}$ the magnetic parameters of the investigated thicker Co film, also grown by molecular beam epitaxy onto oxidized $\mathrm{Si}$ with $\mathrm{Au}$ buffer layer, change more significantly with the number of the traced hysteresis loops, $n$. Also, $\left|H_{E B}\right|$ is slightly larger than the value extrapolated from the dependence of $\left|H_{E B}\right|$ on the FM layer thickness given by Gruyters for $\mathrm{Co} / \mathrm{CoO}(2 \mathrm{~nm})$ bilayers, ${ }^{35}$ which, for a $30 \mathrm{~nm}$ thick Co layer, should be around $251 \mathrm{Oe}$. This difference can be ascribed to several reasons, being one of these the fact that our $\mathrm{CoO}$ is slightly thicker $(3 \mathrm{~nm})$. Usually, $\left|H_{E B}\right|$ increases with the AFM thickness in this regime with low AFM layer thickness. ${ }^{36}$ Moreover, it should also be taken into account that although our sample was grown in a similar fashion, the bilayers of Gruyters were grown on top of $\mathrm{Si}(111)$ substrates with hydrogen passivation, while our sample was deposited on top of a thermally-oxidized $\mathrm{Si}(100)$ substrate which was covered with a $10 \mathrm{~nm}$ thick Au buffer layer. Moreover, the blocking temperature of the samples of Gruyters is around $175 \mathrm{~K}$, whereas the blocking temperature of our $\mathrm{CoO}$ layer is $125 \mathrm{~K}$, suggesting that the formed $\mathrm{CoO}$ might be more hyperstoichiometric with a higher degree of polycrystallinity, i.e., more prone to nanostructuring effects. ${ }^{22,37}$

The best fitting magnetization curves, simulated through the above model, are also plotted in Fig. 1. The respective variations of $t_{i} K_{i}$ and $J_{i}$ used in the simulations are given in Fig. 2. Polarized neutron reflectivity ${ }^{38,39}$ and anisotropic magnetoresistance ${ }^{30}$ studies have shown that, for $\mathrm{Co} / \mathrm{CoO}$ bilayers, the magnetization reversal taking place during the descending branch of the first loop is due to nucleation and motion of domain walls. Since our model considers coherent rotation only, simulations regarding the first loop were not performed. In order to separate the anisotropy contributions 
to $H_{C}$ resulting from the FM and from the rot-type UCSs, we traced the hysteresis loop of a Co film grown under the same conditions as the oxidized one but capped with a $15 \mathrm{~nm}$ thick $\mathrm{Au}$ layer to prevent surface oxidation (not shown). The FM uniaxial anisotropy value estimated for this film is two orders of magnitude lower than those of the UCSs.

In the simulations, we assumed that the FM does not undergo any other modification through the hysteresis loop tracing, except for a change of its easy-axis distribution. Figure 2 shows that neither FM/set nor FM/rot exchange coupling constants depends on $n$. While $t_{\text {rot }} K_{\text {rot }}$ shows a drop between the second and third loops only and remains constant for the subsequent loops, $t_{s e t} K_{s e t}$ shows a steady decrease up to the fourth loop measurement. Due to the low $J_{\text {set }}$ value, however, the changes of $t_{\text {set }} K_{\text {set }}$ do not influence significantly either $H_{C}$ or $H_{E B}$ variations with $n$, as will be discussed in more detail below.

Another parameter that plays an essential role in EB systems is the distribution of the easy-magnetization axes of the involved magnetic phases ${ }^{40}$ characterized by standard deviations $\sigma_{i}$. Such distributions were included in our simulations in order to account for differences in the shape of hysteresis loops. The continuous increase of $\sigma_{\text {rot }}$ with $n$ (see Fig. 2) implies reorientation of some RA easy axes away from that of the cooling field. Conversely, $\sigma_{\text {set }}$ shows a rather small increase between the second and the third magnetic cycles and remains constant for $n>3$. Thus, given that the coupling parameters $t_{\text {set }} K_{\text {set }}$ and $\sigma_{\text {set }}$ barely change with the loop cycling, the coercivity decrease seems to be caused predominantly by the effective spreading of the RA easy-axis distribution with $n$. This may result from magnetic exchange and/or dipolar interactions between neighboring interface UCSs not taken into account in the present model. For example, at the remanence achieved after the first positive field saturation, all rot-type UCSs lay in the vicinity of their easy anisotropy directions established during the EB setting process. At remanence after subsequent negative saturation, however, some of these UCSs, due to coupling with stable neighboring spins, assume new, different minima orientations closer to the easy axes of their stable neighbors. Subsequent hysteresis loop cycling does not change these configurations. For more details about this mechanism, we refer to Ref. 16.

Representative AMR fitting curves for two magnetoresistance cycles in Fig. 3 were calculated using the same parameters employed in the corresponding magnetization simulations. Although the agreement between the experiment and model appears to a certain extent compromised by the measurement noise, the simulated peaks' positions and their magnitude practically coincide with the measured ones. Thus, we consider that these fittings support the validity of our model, which succeeds to reproduce the experiment with no need to assume coexisting positive and negative coupling between UCSs. ${ }^{41}$

Figure 2 shows that while the products of $t_{\text {rot }}$ and $K_{\text {rot }}$ are smaller than $t_{\text {set }} K_{\text {set }}$, the estimated $J_{r o t}$ is always higher than the corresponding $J_{\text {set }}$, contradicting the traditional understanding that the anisotropy and exchange coupling of the interfacial RA grains are weaker than those of the biasing

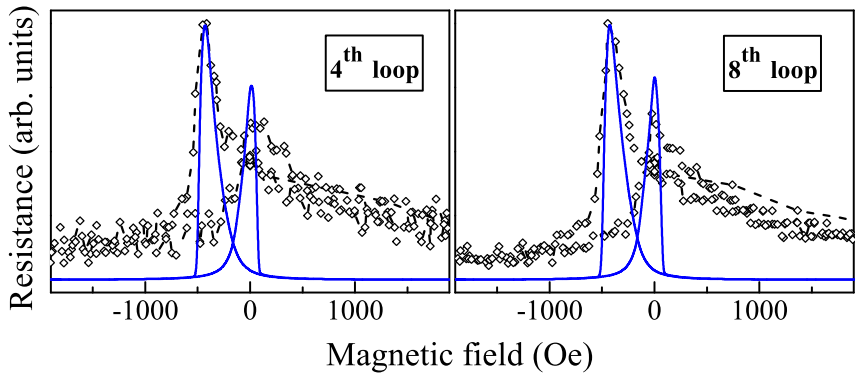

FIG. 3. Anisotropic magnetoresistance data corresponding to the fourth and eighth easy-axis loops. The experimental data are represented by the symbols, and the solid lines are fitting curves, obtained employing the same parameters as for the respective magnetization simulations. The dashed lines are guides to the eye.

spins. ${ }^{4}$ However, it has been reported that thin $\mathrm{CoO}$ films with low magnetocrystalline anisotropy can have large rotatable anisotropy. ${ }^{36,43}$ As mentioned above, the classification of the interface UCSs has been recently examined, ${ }^{23}$ and it has been demonstrated that although highly anisotropic, an UCS can contribute to $H_{C}$ and not to $H_{E B}$ if it is strongly coupled to the adjacent FM. Conversely, a low-anisotropy UCS will add to EB if the coupling to the FM is sufficiently weak. Moreover, provided that the FM/UCS coupling is higher than a certain value, an UCS is rotatable, independent of its anisotropy strength. Our results confirm the above assertions.

There still exist controversies concerning the origin of the interface UCSs. Berkowitz et al. ${ }^{24}$ have found strong indications of a hard Co-ferrite-like phase at the permalloy/ $\mathrm{CoO}$ interface resulting from chemical reactions; for $\mathrm{IrMn} /$ CoFe systems, O'Grady et al. ${ }^{25}$ have inferred that the UCSs are AFM spins. Given that our experiments do not provide atomic level information about the microstructure and chemistry of the interfacial region between $\mathrm{Co}$ and $\mathrm{CoO}$, we are unable to determine the origin of the interface UCSs in our films. We may consider two possibilities. First, all grains with UCSs may have similar shapes and originate from one magnetic phase only (e.g., the AFM), characterized by $K_{\text {rot }}=K_{\text {set }}$. In this case, one obtains from Fig. 2 that $v_{\text {set }}>v_{\text {rot }}$. Alternatively, the set- and rot-type UCSs may represent different magnetic phases and may have different shapes. Then, from the $t_{i} K_{i}$ data in Fig. 2, one obtains that if $v_{\text {set }} \geq 1.2 v_{\text {rot }}, K_{\text {rot }}$ would be higher than $K_{\text {set }}$ for all $n$. This means that rot-type UCSs that are strongly coupled to the UCSs can also be magnetically harder than the set-type UCSs, again confirming the theoretical predictions. ${ }^{23}$

Binek $^{18}$ derived an implicit sequence equation that determines the training effect in conventional EB systems $H_{E B}(n+1)$ from its predecessor, $H_{E B}(n)$,

$$
\left|H_{E B}(n+1)\right|=\left|H_{E B}(n)\right|-\gamma\left(\left|H_{E B}(n)\right|-\left|H_{E B}(\infty)\right|\right)^{3},
$$

using nonequilibrium thermodynamics and considering that consecutive cycling of the FM triggers the spin configurational relaxation at the interface towards equilibrium. In the above equation, $\gamma$ is a temperature-dependent parameter, ${ }^{42}$ and $H_{E B}(\infty)$ is the equilibrium EB field. We used the training effect between the second and the third loops to yield $\gamma=1.18 \times 10^{-4}$ and $H_{E B}(\infty)=183 \mathrm{Oe}$, which in turn were 


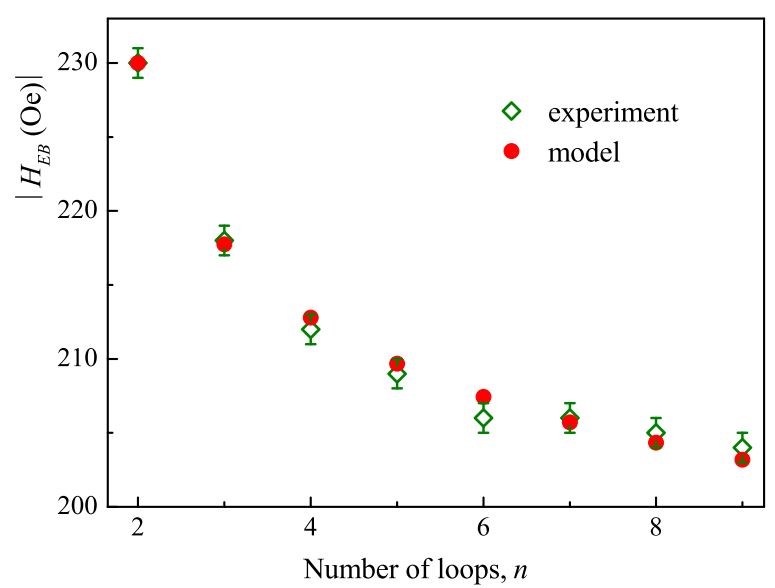

FIG. 4. Training effect of $\left|H_{E B}\right|$ versus $n$ for $n>1$. The solid circles represent the fitting data calculated through $\left|H_{E B}(n+1)\right|=\left|H_{E B}(n)\right|-$ $\gamma\left(\left|H_{E B}(n)\right|-\left|H_{E B}(\infty)\right|\right)^{3}$ using $\gamma=1.18 \times 10^{-4}$ and $H_{E B}(\infty)=183$ Oe.

used to obtain the theoretical data from Eq. (1). As can be seen in Fig. 4, there is a good agreement between the model and experiment, which seems to support training related to irreversible reconfigurations at the FM/AFM interface that is scaling with $n .^{36,43,44}$

\section{SUMMARY}

We investigated the low-temperature exchange-bias training effect in a $\mathrm{Co} / \mathrm{CoO}$ film. Numerical simulations were performed through a polycrystalline model for exchange bias from which the magnetic parameters of the ferromagnet and of the interfacial uncompensated spins were extracted. The good agreement between experiment and simulation confirms previous experimental evidence that inhomogeneous though coherent magnetization rotation is the dominant reversal mechanism in this system. We obtain that the exchange coupling between the ferromagnetic layer and the interfacial rotatable uncompensated spins is stronger than the coupling between the ferromagnet and the spins responsible for the bias. We also estimate that the training is caused predominantly by variations of the rotatable anisotropy parameters. Our results confirm recent theoretical predictions that uncompensated spins strongly coupled to the ferromagnet can contribute to the coercivity rather than to the bias, no matter what their anisotropy strength is. Further investigations are required to verify whether these characteristics are specific for our $\mathrm{Co} / \mathrm{CoO}$ films only or are valid for other exchange-bias systems as well.

\section{ACKNOWLEDGMENTS}

This work has been supported by the Research FoundationFlanders (FWO), the KU Leuven Concerted Action (GOA/09/ 006 and GOA/14/007) programs, and the Brazilian foundation CNPq under Project Nos. 307082/2012-1, 483277/2012-6, and 245897/2012-7. We appreciate useful discussions with A. Harres and J. Nogués. E.M. thanks the FWO for financial support.

${ }^{1}$ W. H. Meiklejohn and C. P. Bean, Phys. Rev. 102, 1413 (1956); W. H. Meiklejohn and C. P. Bean, Phys. Rev. 105, 904 (1957).
${ }^{2}$ J. Nogués and I. K. Schuller, J. Magn. Magn. Mater. 192, 203 (1999).

${ }^{3}$ A. E. Berkowitz and K. Takano, J. Magn. Magn. Mater. 200, 552 (1999).

${ }^{4} J$. Nogués, J. Sort, V. Langlais, V. Skumryev, S. Suriñach, J. S. Muñoz, and M. D. Baró, Phys. Rep. 422, 65 (2005).

${ }^{5}$ V. Skumryev, S. Stoyanov, Y. Zhang, G. Hadjipanayis, D. Givord, and J. Nogués, Nature 423, 850 (2003).

${ }^{6}$ J. Fassbender, D. Ravelosona, and Y. Samson, J. Phys. D: Appl. Phys. 37, R179 (2004); J. Fassbender and J. McCord, J. Magn. Magn. Mater. 320, 579 (2008).

${ }^{7}$ D. Schafer, J. Geshev, S. Nicolodi, L. G. Pereira, J. E. Schmidt, and P. L. Grande, Appl. Phys. Lett. 93, 042501 (2008); D. Schafer, P. L. Grande, L. G. Pereira, and J. Geshev, J. Appl. Phys. 109, 023905 (2011).

${ }^{8}$ J. Nogués, L. Morellon, C. Leighton, M. R. Ibarra, and I. K. Schuller, Phys. Rev. B 61, R6455 (2000).

${ }^{9}$ J. Nogués, J. Sort, S. Suriñach, J. S. Muñoz, M. D. Baró, J. F. Bobo, U. Lüders, E. Haanappel, M. R. Fitzsimmons, A. Hoffmann, and J. W. Cai, Appl. Phys. Lett. 82, 3044 (2003).

${ }^{10} \mathrm{P}$. Miltényi, M. Gierlings, M. Bamming, U. May, G. Güntherodt, J. Nogués, M. Gruyters, C. Leighton, and Ivan K. Schuller, Appl. Phys. Lett. 75, 2304 (1999); N. J. Gökemeijer and C. L. Chien, J. Appl. Phys. 85, 5516 (1999); J. Geshev, L. G. Pereira, and V. Skumryev, Phys. Rev. Lett. 100, 039701 (2008).

${ }^{11}$ D. Paccard, C. Schlenker, O. Massenet, R. Montmory, and A. Yelon, Phys. Status Solidi B 16, 301 (1966).

${ }^{12}$ A. G. Biternas, U. Nowak, and R. W. Chantrell, Phys. Rev. B 80, 134419 (2009).

${ }^{13}$ L. E. Fernandez-Outon, G. Vallejo-Fernandez, S. Manzoor, and K. O’Grady, J. Magn. Magn. Mater. 303, 296 (2006).

${ }^{14}$ A. Hoffmann, Phys. Rev. Lett. 93, 097203 (2004).

${ }^{15} \mathrm{~S}$. Brems, K. Temst, and C. Van Haesendonck, Phys. Rev. Lett. 99, 067201 (2007).

${ }^{16}$ A. Harres and J. Geshev, J. Phys.: Condens. Matter 23, 216003 (2011).

${ }^{17}$ L. Néel, Ann. Phys. 2, 61 (1967).

${ }^{18}$ C. Binek, Phys. Rev. B 70, 014421 (2004).

${ }^{19}$ D. Suess, M. Kirschner, T. Schrefl, and J. Fidler, Phys. Rev. B 67, 054419 (2003).

${ }^{20}$ A. G. Biternas, R. W. Chantrell, and U. Nowak, Phys. Rev. B 82, 134426 (2010).

${ }^{21}$ P. Miltényi, M. Gierlings, J. Keller, B. Beschoten, G. Güntherodt, U. Nowak, and K. D. Usadel, Phys. Rev. Lett. 84, 4224 (2000); U. Nowak, Phys. Rev. B 66, 014430 (2002).

${ }^{22}$ E. Menéndez, J. Demeter, J. Van Eyken, P. Nawrocki, E. Jedryka, M. Wójcik, J. F. Lopez-Barbera, J. Nogués, A. Vantomme, and K. Temst, ACS Appl. Mater. Interfaces 5, 4320 (2013).

${ }^{23}$ A. Harres and J. Geshev, J. Phys.: Condens. Matter 24, 326004 (2012).

${ }^{24}$ A. E. Berkowitz, J.-I. Hong, S. K. McCall, E. Shipton, K. T. Chan, T. Leo, and D. J. Smith, Phys. Rev. B 81, 134404 (2010).

${ }^{25}$ K. O'Grady, L. E. Fernandez-Outon, and G. Vallejo-Fernandez, J. Magn. Magn. Mater. 322, 883 (2010).

${ }^{26}$ R. D. McMichael, M. D. Stiles, P. J. Chen, and W. F. Egelhoff, Jr., Phys. Rev. B 58, 8605 (1998).

${ }^{27}$ J. Geshev, L. G. Pereira, and J. E. Schmidt, Phys. Rev. B 66, 134432 (2002).

${ }^{28}$ Z. Li and S. Zhang, Phys. Rev. B 61, R14897 (2000).

${ }^{29}$ E. Fulcomer and S. Charap, J. Appl. Phys. 43, 4190 (1972).

${ }^{30} \mathrm{~S}$. Brems, D. Buntinx, K. Temst, C. Van Haesendonck, F. Radu, and H. Zabel, Phys. Rev. Lett. 95, 157202 (2005).

${ }^{31}$ J. Geshev, A. D. C. Viegas, and J. E. Schmidt, J. Appl. Phys. 84, 1488 (1998).

${ }^{32}$ W. L. Roth, Phys. Rev. 110, 1333 (1958).

${ }^{33}$ W. Jauch, M. Reehuis, H. J. Bleif, F. Kubanek, and P. Pattison, Phys. Rev. B 64, 052102 (2001).

${ }^{34}$ A. Schrön, C. Rödl, and F. Bechestedt, Phys. Rev. B 86, 115134 (2012).

${ }^{35}$ M. Gruyters, Europhys. Lett. 64, 803 (2003).

${ }^{36}$ T. Gredig, I. N. Krivorotov, and E. D. Dahlberg, J. Appl. Phys. 91, 7760 (2002).

${ }^{37}$ G. Nowak, A. Remhof, F. Radu, A. Nefedov, H.-W. Becker, and H. Zabel, Phys. Rev. B 75, 174405 (2007)

${ }^{38}$ F. Radu, M. Etzkorn, R. Siebrecht, T. Schmitte, K. Westerholt, and H. Zabel, Phys. Rev. B 67, 134409 (2003).

${ }^{39}$ J. Demeter, E. Menéndez, A. Teichert, R. Steitz, D. Paramanik, C. Van Haesendonck, A. Vantomme, and K. Temst, Solid State Commun. 152, 292 (2012). 
${ }^{40}$ J. Geshev, S. Nicolodi, L. G. Pereira, J. E. Schmidt, V. Skumryev, S. Suriñach, and M. D. Baró, Phys. Rev. B 77, 132407 (2008).

${ }^{41}$ K. Zhang, T. Zhao, and H. Fujiwara, J. Appl. Phys. 89, 6910 (2001).

${ }^{42}$ C. Binek, X. He, and S. Polisetty, Phys. Rev. B 72, 054408 (2005).
${ }^{43}$ T. Gredig, I. N. Krivorotov, and E. D. Dahlberg, Phys. Rev. B 74, 094431 (2006).

${ }^{44}$ F. Radu and H. Zabel, Springer Tracts in Modern Physics (Springer, 2008), Vol. 227, p. 97. 